\title{
The Prevalence and Social Determinants of Hypertension among Adults in Indonesia: A Cross-Sectional Population-Based National Survey
}

\author{
Karl Peltzer (iD) ${ }^{1,2}$ and Supa Pengpid ${ }^{2,3}$ \\ ${ }^{1}$ HIV/AIDS/STIs and TB (HAST), Human Sciences Research Council, Pretoria 0001, South Africa \\ ${ }^{2}$ Department of Research and Innovation, University of Limpopo, Sovenga 0727, South Africa \\ ${ }^{3}$ ASEAN Institute for Health Development, Mahidol University, Salaya, Thailand \\ Correspondence should be addressed to Karl Peltzer; karl.peltzer@tdt.edu.vn
}

Received 5 January 2018; Accepted 2 August 2018; Published 9 August 2018

Academic Editor: Claudio Borghi

Copyright (C) 2018 Karl Peltzer and Supa Pengpid. This is an open access article distributed under the Creative Commons Attribution License, which permits unrestricted use, distribution, and reproduction in any medium, provided the original work is properly cited.

\begin{abstract}
Background. Hypertension is the most significant avoidable cause of morbidity and mortality, yet nationally representative adult data on Indonesia have not been available. The study aimed at assessing the prevalence and determinants of hypertension, including sociodemographic variables, weight status, health behaviour, and psychosocial stress and support risk factors. Methods. The Indonesia Family Life Survey (IFLS-5) interviewed and examined in a national population-based cross-sectional study 29965 individuals aged 18 years and older, mean age 43.3 years $(\mathrm{SD}=15.3)$. Blood pressure, body height and weight, dietary behaviour, physical activity, tobacco use, and psychosocial variables were measured. Logistic regression analyses were used to estimate determinants of hypertension by gender. Results. The prevalence of hypertension among study participants was $33.4 \%$ (95\% CI: 32.7-34.0), among males 31.0\% (95\% CI: 30.2, 31.9), and among females 35.4\% (95\% CI: 34.6, 36.3). Among hypertensives, $42.9 \%$ were aware, $11.5 \%$ were treated, and $14.3 \%$ were controlled. In fully adjusted analyses, in both men and women, older age, no or elementary education, being overweight or obese, and having visited an outpatients health facility in the past 4 weeks were positively associated hypertension. Significant linear relationships of hypertension were found with age $(P$ for trend $<0.001)$ and body mass index $(\mathrm{BMI})(P$ for trend $<0.001)$. In addition, among men having quit tobacco use and depressive symptoms were positively associated with hypertension, while current tobacco use was negatively associated with hypertension. Moreover, among women lower subjective economic status was associated with hypertension. Conclusions. The prevalence of hypertension was high and awareness was low, and treatment and control were very low. Significant multilevel public health interventions are urgently needed to improve the diagnosis, treatment, and control of hypertension in Indonesia.
\end{abstract}

\section{Introduction}

Chronic noncommunicable diseases (NCDs) have become an enormous health burden in low- and middle-income countries [1]. The scale of the increase of NCDs can be particularly observed in Southeast Asia, such as in Indonesia, where a limited budget has been allocated to combat the growing NCD epidemic [2]. The three top causes of death in Indonesia are stroke (21.2\%), ischaemic heart disease (8.9\%), and diabetes mellitus (6.5\%) [3]. Major adult NCD risk factors in Indonesia include current tobacco smoking (in 2011) 35\% (67\% in men and 3\% in women), high blood pressure or hypertension (in 2008) $27.8 \%$ (29.1\% in men and
$26.5 \%$ in women), and obesity (in 2008) $4.8 \%$ (2.6\% in men and $6.0 \%$ in women) [4].

Hypertension is a preventable illness condition and has been found to be associated with an unhealthy lifestyle, including tobacco smoking, a lack of physical activity, and alcohol consumption [1]. Increasing trends in the prevalence of hypertension have been shown in high- and low- and middle-income countries $[5,6]$. Comparable with the global trend, "the single most attributable cause for mortality in South-East Asia" is hypertension with an increasing trend [7]. Hussain et al. [8] found in the "2007 Indonesian Family Life Survey (IFLS-4)" a prevalence of $47.8 \%$ with hypertension in individuals 40 years and older. In other national 
population-based surveys in Southeast Asia the prevalence of hypertension was in Malaysia 43.5\% ( $\geq 30$ years in 2011) [9], in Myanmar 30.1\% (15-64-year-olds in 2009) [10], in Sri Lanka $23.7 \%$ ( $\geq 18$ years in 2005-2006) [11], and in Vietnam $25.1 \%$ ( $\geq 25$ years in 2002-2008) [12], while in two other Asian countries the national prevalence of hypertension was in China 29.6\% ( $\geq 18$ years in 2010) [13] and in Iran 25\% (25-64year-olds in 2005) [14]. There is a lack of data on the adult (15 years and older) hypertension prevalence in Indonesia.

Hypertension is a preventable disease but recent studies found a low prevalence of antihypertensive medication treatment. In the 2007 IFLS4 survey (40-65 years) in Indonesia, $37 \%$ of individuals with hypertension were diagnosed or aware and $25 \%$ were treated with prescribed antihypertensive medication [8]. In the previously reported national population-based surveys in China ( $\geq 18$ years and older), $42.6 \%$ were aware of their hypertension status and $34.1 \%$ received antihypertensive treatment [13], in Iran (25-64 yearolds) $34 \%$ were aware and $24.8 \%$ treated [14], and in Vietnam [ $\geq 25$ years] $48.4 \%$ were aware and $29.5 \%$ received treatment [12]. In a community study with participants (35-70 years) from 17 mainly low- and middle-income countries, $46.5 \%$ were aware of their hypertension diagnosis and $32.5 \%$ were treated [15]. These studies indicate the very important need for the improvement of the diagnosis and treatment of hypertension [15].

Various risk factors have been found to be linked with hypertension, including sociodemographics (older age, female or male gender, lower education level, and lower household income) [8, 10-12, 14-17], geolocality (urban residence) $[10,12,18]$, and other risk factors, including body weight status and health behaviour and psychosocial stress and support. Higher body mass index (BMI) has been found to be positively $[8,10,13,15-17]$ and underweight status negatively [10] associated with hypertension. Various dietary behaviours were also associated with hypertension, including insufficient fruit and vegetable intake [19-21], consumption of fatty foods $[22,23]$ and fast foods $[24,25]$, and sugar and soft drink consumption [26]. A number of studies found an association between physical inactivity [10, 13, 27-29], smoking [29, 30], and hypertension. Some studies, e.g., [31], found that a higher frequency of doctor visits was associated with having hypertension in addition to psychosocial stress $[32,33]$, including depression [34], low life satisfaction [35, 36], and less social cohesion [37] and lack of social support [32].

The aim of this study was to assess the prevalence of hypertension and to identify sociodemographic, weight status, health behaviour, psychosocial stress, and support risk factors for hypertension based on a cross-sectional survey of the IFLS-5 in 2014-15 in Indonesia.

\section{Materials and Methods}

2.1. Study Design and Participants. Data were analysed from the "Indonesia Family Life Survey (IFLS-5)", a continuing demographic and health survey that began in 1993 and had since four rounds of data collection, with the fifth wave (IFLS-5) having been completed in 2015 [38]. The community surveys collected data on household, individual, and community level using a multistage stratified sampling [38]. The sampling frame of the first survey in 1993 was based on households from 321 enumeration areas (EAs) (20 households were randomly selected from each urban EA, and 30 households were selected from each rural EA) in 13 out of 27 Indonesian provinces that were selected representing $83 \%$ of the Indonesian population in 1993; more details are available in [38]. At household level, several randomly selected household members were asked to provide detailed individual information. In the IFLS-5, 16,204 households and 2996518 years and older individuals were interviewed and had complete blood pressure measurements. In the IFLS-5, "the dynasty recontact rate was $92 \%$ and for the individual target households (including split off households as separate) the re-contact rate was $90.5 \%$." [39]. Although the survey is longitudinal, we restricted our analysis to the IFLS- 5 because it was the only survey to include complete hypertension information for persons 18 years and older.

2.2. Measures. Measures included blood pressure measurements, anthropometric measurements, and questionnaire items on sociodemographic factors, dietary behaviour, physical activity, tobacco use, health care utilization, psychosocial stress, and support.

2.2.1. Blood Pressure (BP) Measurements and Classification. Three successive measurements of systolic and diastolic blood pressure (BP) were recorded with an Omron meter, HEM-7203, by regular trained interviewers on household members 18 years and older at home in a seated position [38]. Mostly a normal sized cuff was used, while large cuffs were available when needed [38]. The first BP measurement was conducted at the onset of the research interview, with two subsequent assessments during the course of the interview [38]. Information was collected on awareness (having been diagnosed of hypertension by a doctor, nurse, paramedic, and trained mid-wife) and treatment of hypertension (currently taking prescribed medication on a weekly basis to manage your hypertension). The current analysis is restricted to individuals who had information on hypertension measurement, awareness, and treatment. For the three measurements of each systolic and diastolic blood pressure average blood pressure was calculated arithmetically.

Blood pressure was classified using JNC 7 algorithm [39]. "Hypertension was defined as systolic BP $\geq 140 \mathrm{~mm} \mathrm{Hg}$ and/or DBP $\geq 90 \mathrm{~mm} \mathrm{Hg}$ and/or current use of antihypertensive medication. Normotension was defined as BP values $<120 / 80$ $\mathrm{mm} \mathrm{Hg}$ in individuals who were not taking antihypertensive medication" [39].

Sociodemographic factors were assessed with questions on age, gender, education, residential status, subjective socioeconomic background, and country region. Subjective economic status was assessed by the question "Please imagine a sixstep ladder where on the bottom (the first step), stand the poorest people, and on the highest step (the sixth step), stand the richest people. On which [economic] step are you today?" The answers ranged from (1) poorest to (6) richest [38]. 


\subsubsection{Weight Status and Health Behaviour}

Anthropometric Measurements. Heights were measured to the nearest millimetre using a Seca plastic height board (model 213) [38]. Weights were taken to the nearest tenth of a kilogram using a Camry model EB1003 scale [38]. Body mass index (BMI) was calculated as weight in $\mathrm{kg}$ divided by height in metre squared and classified according to Asian criteria: normal weight $\left(18.5\right.$ to $\left.<23.0 \mathrm{~kg} / \mathrm{m}^{2}\right)$, overweight $(23.0$ to $\left.<25.0 \mathrm{~kg} / \mathrm{m}^{2}\right)$ and obese $\left(25+\mathrm{kg} / \mathrm{m}^{2}\right)[40]$.

Vegetable consumption was measured with two items, "How many days in the past week, (1) Green leafy vegetables and (2) carrots had been eaten?" [38].

Fruit consumption was assessed with three questions on, "How many days in the past week, (1) Banana, (2) Mango and (3) Papaya had been eaten?" [38].

Additional dietary variables included "on how many days in the past week, (1) Fast food, (2) Fried snacks (tempe, tahu, bakwan, etc.), and (3) Soft drinks (Coca cola, sprite, etc.) had been consumed?" [38].

Physical Activity. Physical activity was assessed using a modified version of the International Physical Activity Questionnaire (IPAQ) short version, for the last 7 days (IPAQ-S7S). We used the instructions given in the IPAQ manual [41] and categorized physical activity according to the official IPAQ scoring protocol [42] as low, moderate, and high.

Tobacco use was assessed with two questions: (1) "Have you ever chewed tobacco, smoked a pipe, smoked selfenrolled cigarettes, or smoked cigarettes/cigars?" (Yes, No) and (2) "Do you still have the habit or have you totally quit?"(Still have, Quit) [38]. Responses were grouped into never, quitters, and current tobacco users.

Health care utilization was assessed with the question, "Whether visited any outpatient health care clinic in one month prior to survey or not?" (Yes, No) [38].

2.2.3. Psychosocial Stress and Support. The Centres for Epidemiologic Studies Depression Scale (CES-D: 10 items) was used to assess depressive symptoms, and scores 10 or more were classified as having depressive symptoms [43] (Cronbach's alpha $=0.71$.

Life satisfaction was assessed with one item, "Please, think about your life as a whole. How satisfied are you with it?" Response option ranged from 1: completely satisfied to 5: not at all satisfied [38].

Social support or capital was assessed with 4 items (selected from 11 items through factor analysis), "During the last 12 months did you participate or use?..." (1) Community meeting, (2) Voluntary labour, (3), Programme to improve the village/neighbourhood, and (4) Religious activities. Response options were "yes" or "no" [38] (Cronbach's alpha 0.60).

2.3. Data Analysis. Descriptive statistics were used to describe the study variables of the study population and Chi-square test to calculate difference in proportions. Nonparametric tests were used for trend analyses across ordered groups. Logistic regression (forced entry) analyses were used to test significant determinants of hypertension status, with hypertension serving as the dichotomous outcome variable and in Model 1 age served as independent predictor variable and in Model 2 sociodemographics, health, nutrition, psychosocial stress, and social capital served as the independent predictor variables. We also examined linear associations by exposure categories in each model ( $p$ for trend) in the assessment of hypertension prevalence. "Cross-section analysis weights were applied to correct both for sample attrition from 1993 to 2014, and then to correct for the fact that the IFLS1 sample design included over-sampling in urban areas and off Java. The cross-section weights are matched to the 2014 Indonesian population, again in the 13 IFLS provinces, in order to make the attrition-adjusted IFLS sample representative of the 2014 Indonesian population in those provinces"[38]. Both the 95\% confidence intervals and $\mathrm{P}$ values were adjusted considering the survey design of the study. All analyses were conducted with STATA software version 13.0 (Stata Corporation, College Station, TX, USA).

\section{Results}

3.1. Sample Characteristics. The total sample included 29965 individuals 18 years and older, with complete hypertension measures (females: 52.7\%; mean age 43.3 years, SD: 15.3 , age range of 18-109 years) from Indonesia. Overall, $33.4 \%$ of the population had hypertension, $31.0 \%$ among men and $35.4 \%$ among women. Among hypertensives $42.9 \%$ were aware that they had hypertension, which was significantly higher in women $(50.1 \%)$ than men $(33.7 \%)$ and in urban $(44.5 \%)$ than rural areas $(41.1 \%)$. Of the population with hypertension, $11.5 \%$ were currently using prescribed antihypertensive medication, and $14.3 \%$ had controlled their blood pressure ( $<140 \mathrm{~mm} \mathrm{Hg}$ and diastolic blood pressure $<90 \mathrm{~mm} \mathrm{Hg}$ ). Women $(14.0 \%)$ were more often than men $(8.4 \%)$ and urban dwellers (13.0\%) more often than rural dwellers (9.9\%) using currently prescribed antihypertensive medication. Of those participants with hypertension, only $14.3 \%$ controlled their blood pressure. Mean systolic blood pressure was $1.1 \mathrm{mmHg}$ higher for men than for women, while mean diastolic blood pressure was $0.2 \mathrm{mmHg}$ higher for women than for men. The prevalence of BMI obesity was higher in women (42.0\%) than in men $(23.0 \%)$, while the current tobacco use prevalence was significantly higher in men $(67.2 \%)$ than in women $(3.0 \%)$ (see Table 1).

The prevalence of hypertension increases overall and for both sexes by age group from 18 to 29 years to 70 years and above ( $P$ for trend $<0.001$ ). The prevalence of hypertension is higher among males than females at the age 18 to 29 years, while from 40 years and older the prevalence of hypertension is higher among women than men. Further the prevalence of hypertension decreases overall and for both sexes (and stronger for females) by education from having no education to having higher education $(P$ for trend $<0.001)$ (see Table 2$)$.

\section{Association between Psychosocial Risk Factors and Hypertension}

Table 3 shows associations (odds ratios) between independent variables and the prevalence of hypertension, in Model 1 
TABLE 1: Sample characteristics.

\begin{tabular}{|c|c|c|c|c|c|}
\hline Variable name & Variable specification & Total & Male & Female & P value \\
\hline Sample & $\mathrm{N}(\%)$ & 29965 & $13997(47.3)$ & $15969(52.7)$ & \\
\hline Mean age in years (SD) & $\begin{array}{c}\text { Range } 18-109 \text {, mean } \\
(\mathrm{SD})\end{array}$ & $43.3(15.3)$ & $42.0(15.3)$ & $42.6(15.2)$ & 0.056 \\
\hline Systolic blood pressure & mmHg, mean (SD) & $130.9(21.9)$ & $131.8(19.2)$ & $130.1(24.1)$ & $<0.001$ \\
\hline Diastolic blood pressure & mmHg, mean (SD) & $80.1(11.9)$ & $80.0(11.7)$ & $80.2(12.1)$ & 0.004 \\
\hline Hypertension & $\%$ & 33.4 & 31.0 & 35.4 & $<0.001$ \\
\hline Of hypertensives & Aware, $\%$ & 42.9 & 33.7 & 50.1 & $<0.001$ \\
\hline Of hypertensives & Treated, $\%$ & 11.5 & 8.4 & 14.0 & $<0.001$ \\
\hline Of hypertensives & Controlled, $\%$ & 14.3 & 12.4 & 15.7 & $<0.001$ \\
\hline Education & None/elementary, \% & 44.0 & 39.1 & 48.4 & $<0.001$ \\
\hline $\begin{array}{l}\text { Subjective economic } \\
\text { background }\end{array}$ & $\begin{array}{c}\text { Poor, \% } \\
\text { Medium, \% } \\
\text { Rich, \% } \\
\end{array}$ & $\begin{array}{l}25.5 \\
46.6 \\
27.9 \\
\end{array}$ & $\begin{array}{l}27.4 \\
48.9 \\
23.7 \\
\end{array}$ & $\begin{array}{l}23.8 \\
44.6 \\
31.6 \\
\end{array}$ & $<0.001$ \\
\hline Residence & Urban, $\%$ & 58.6 & 58.6 & 58.7 & 0.198 \\
\hline Region & $\begin{array}{c}\text { Sumatra, } \% \\
\text { Java, } \% \\
\text { Main islands }{ }^{1}, \% \\
\end{array}$ & $\begin{array}{l}22.7 \\
54.6 \\
22.7 \\
\end{array}$ & $\begin{array}{l}23.4 \\
54.2 \\
22.4 \\
\end{array}$ & $\begin{array}{l}22.1 \\
54.9 \\
23.0 \\
\end{array}$ & 0.027 \\
\hline \multicolumn{6}{|l|}{$\begin{array}{l}\text { Body weight status and } \\
\text { health behaviour }\end{array}$} \\
\hline Body Mass Index (BMI) & $\begin{array}{c}\text { Normal, \% } \\
\text { Underweight, \% } \\
\text { Overweight, \% } \\
\text { Obese, \% } \\
\end{array}$ & $\begin{array}{l}40.2 \\
11.2 \\
16.0 \\
32.4 \\
\end{array}$ & $\begin{array}{l}48.3 \\
13.5 \\
15.2 \\
23.0 \\
\end{array}$ & $\begin{array}{c}33.2 \\
9.0 \\
16.8 \\
42.0 \\
\end{array}$ & $<0.001$ \\
\hline Vegetable consumption $^{2}$ & $<7$ days/week, $\%$ & 57.6 & 58.3 & 56.7 & $<0.001$ \\
\hline Fruit consumption $^{3}$ & $<3$ days/week, $\%$ & 58.1 & 59.6 & 56.8 & $<0.001$ \\
\hline Fast food & $\geq$ once week, $\%$ & 10.6 & 9.8 & 11.2 & 0.033 \\
\hline Fried snacks & 4-7 times/week, $\%$ & 25.6 & 26.3 & 25.3 & 0.147 \\
\hline Soft drinks & $\geq$ once week, $\%$ & 18.5 & 24.9 & 12.8 & $<0.001$ \\
\hline Physical activity & $\begin{array}{c}\text { Low, \% } \\
\text { Moderate, \% } \\
\text { High, } \% \\
\end{array}$ & $\begin{array}{l}48.3 \\
26.6 \\
25.1 \\
\end{array}$ & $\begin{array}{l}43.1 \\
22.9 \\
34.0 \\
\end{array}$ & $\begin{array}{l}49.7 \\
30.0 \\
20.3 \\
\end{array}$ & $<0.001$ \\
\hline Tobacco use & $\begin{array}{c}\text { Never, \% } \\
\text { Quit, \% } \\
\text { Current, \% } \\
\end{array}$ & $\begin{array}{c}62.2 \\
5.0 \\
32.8 \\
\end{array}$ & $\begin{array}{c}23.2 \\
9.6 \\
67.2 \\
\end{array}$ & $\begin{array}{c}95.9 \\
1.1 \\
3.0 \\
\end{array}$ & $<0.001$ \\
\hline Health care utilization & Past 4 weeks, $\%$ & 18.8 & 14.6 & 23.9 & $<0.001$ \\
\hline \multicolumn{6}{|l|}{$\begin{array}{l}\text { Psychosocial stress and } \\
\text { support }\end{array}$} \\
\hline Depression symptoms & Scores $\geq 10, \%$ & 22.8 & 22.2 & 23.4 & 0.177 \\
\hline Life satisfaction & High-yes, $\%$ & 42.7 & 40.2 & 44.9 & $<0.001$ \\
\hline Social support/capital & High-yes, \% & 37.9 & 50.6 & 26.4 & $<0.001$ \\
\hline
\end{tabular}

adjusted for age, and in Model 2 adjusted for all covariates in the table. In fully adjusted models in both men and women, older age, no or elementary education, being overweight or obese, and having visited an outpatients health facility in the past 4 weeks were positively associated with hypertension. Both among men and among women being underweight was negatively associated with hypertension. In addition, among men having quit tobacco use and depressive symptoms were positively associated with hypertension, while current tobacco use was negatively associated with hypertension. Moreover, among women lower subjective economic status was associated with hypertension. In Model 1, the prevalence of hypertension was higher among men in urban than rural areas, while this became insignificant in Model 2, the fully adjusted model. Significant positive linear relationships of hypertension were found among both men and women with 
TABLE 2: Prevalence of hypertension among individuals 18 years and older in Indonesia.

\begin{tabular}{|c|c|c|c|c|c|c|c|}
\hline \multirow[t]{2}{*}{ Variable } & \multirow{2}{*}{$\begin{array}{c}\text { Total sample } \\
\mathrm{N}\end{array}$} & \multicolumn{2}{|r|}{ Male } & \multicolumn{2}{|r|}{ Female } & \multicolumn{2}{|r|}{ Total } \\
\hline & & $\mathrm{n}$ & $\%(95 \% \mathrm{CI})$ & $\mathrm{N}$ & $\%(95 \% \mathrm{CI})$ & $\mathrm{N}$ & $\%(95 \% \mathrm{CI})$ \\
\hline All & 29965 & 4040 & $31.0(30.2,31.9)$ & 4925 & $35.4(34.6,36.3)$ & 8965 & $33.4(32.7,34.0)$ \\
\hline \multicolumn{8}{|l|}{ Age group } \\
\hline $18-29$ & 8488 & 535 & $13.9(12.8,15.2)$ & 433 & $9.3(8.4,10.2)$ & 968 & $11.6(10.9,12.4)$ \\
\hline $30-39$ & 8444 & 804 & $19.2(17.8,20.5)$ & 834 & $19.5(18.2,20.9)$ & 1638 & $19.3(18.4,20.3)$ \\
\hline $40-49$ & 5586 & 874 & $31.3(29.5,33.2)$ & 1093 & $39.1(37.2,41.1)$ & 1967 & $35.6(34.3,37.0)$ \\
\hline $50-59$ & 3834 & 809 & $45.6(43.1,48.2)$ & 1167 & $56.7(54.4,59.0)$ & 1967 & $51.3(49.5,53.0)$ \\
\hline $60-69$ & 2105 & 582 & $58.1(54.7,61.4)$ & 730 & $66.0(62.8,69.0)$ & 1312 & $62.1(59.8,64.4)$ \\
\hline \multirow[t]{2}{*}{70 or more } & 1508 & 436 & $67.1(63.1,70.9)$ & 668 & $79.3(76.0,82.3)$ & 1104 & $74.1(71.5,76.4)$ \\
\hline & & & $P$ - trend $<0.001$ & & $P$ - trend $<0.001$ & & $P$ - trend $<0.001$ \\
\hline \multicolumn{8}{|l|}{ Education } \\
\hline None & 1568 & 200 & $47.2(41.8,52.6)$ & 699 & $59.3(56.0,62.5)$ & 899 & $56.1(53.2,58.8)$ \\
\hline Elementary & 9741 & 1525 & $36.8(35.2,38.4)$ & 2292 & $45.2(43.8,46.7)$ & 3817 & $41.5(40.4,42.6)$ \\
\hline High school & 14305 & 1736 & $25.7(24.6,26.9)$ & 1537 & $24.6(23.5,25.8)$ & 3273 & $25.2(24.4,26.0)$ \\
\hline \multirow[t]{2}{*}{ Higher education } & 4294 & 567 & $30.1(27.9,32.5)$ & 365 & $20.0(18.1,22.1)$ & 932 & $25.1(23.4,26.6)$ \\
\hline & & & $P$ - trend $<0.001$ & & $P$ - trend $<0.001$ & & $P$ - trend $<0.001$ \\
\hline
\end{tabular}

age $(\mathrm{P}<0.001)$ and $\mathrm{BMI}(\mathrm{P}<0.001)$, and negative relationships among men with tobacco use $(\mathrm{P}=0.006)$ and among women with economic background $(\mathrm{P}=0.015)$ (see Table 3$)$.

\section{Discussion}

In this first nationally representative population-based survey on hypertension in Indonesia, the age-adjusted prevalence in all adults (18 years and older) was $33.4 \%$ (48.6\% in 40 years and older) in 2014-15, which is similar to the prevalence among 40 years and older in the 2007 survey in Indonesia (47.8\%) [8], the Myanmar survey in 2009 of $30.1 \%$ in 15 64-year-olds [10], and the China survey in 2010 of $29.6 \%$ in individuals 18 years and older [13], and similar to the global adult ( $\geq 20$ years) prevalence in low- and middle-income countries (31.5\%) [44], but higher than in Iran [14, 45], Sri Lanka [11], and Vietnam [12].

Of those who had hypertension only $42.9 \%$ were aware (44.9\% among 40 years and older) and $11.5 \%$ were using prescribed antihypertensive medication (13.8\% among 40 years and older) in this 2014-15 survey. This seems to show an increase in the awareness (from $37 \%$ in 2007 to $44.9 \%$ in 2014 15 ) but a decrease in the proportion of treatment (from $25 \%$ in 2007 to $11.5 \%$ in 2014-15) [8]. The proportion of awareness in this study was also similar to figures reported from China [13], Vietnam [12], and globally in low- and middle-income countries $[15,45]$; however, the proportion of reported treatment was in this study significantly lower than in other countries, including China [13], Iran [14], Vietnam [12], and globally in low- and middle-income countries studies $[15,45]$. The awareness and treatment of hypertension was in this study greater among women than men, as also found previously [8]. The awareness and treatment of hypertension was higher in urban than rural areas. This may be because of lack of access to health care services in rural areas [8]. The increase in the awareness of hypertension may be attributed to communitybased integrated coaching posts that enable community participation in the early detection of hypertension [46]. These findings seem to suggest that despite some improvement in the awareness of hypertension more should be achieved, in particular among men, and appropriate treatment needs to be seriously improved, especially also among men and in rural areas.

This study found, in agreement with various studies [8, 10-12, 14-17], that older age and lower educational level were among both men and women associated with hypertension. Lower subjective economic status was among women but not among men associated with hypertension. Although the prevalence of hypertension increased overall and for both sexes by age group from 18 to 29 years to 70 years and above, the prevalence of hypertension was higher among males than females at the age 18 to 29 years, while from 40 years and older the prevalence of hypertension was higher among women than men. One possible reason for this age differential among the sexes can be because the prevalence rates of obesity, as one important risk factor for hypertension, are higher in boys than girls among children, adolescents, and young adults [47-49] but higher in females in the adult age groups [49]. The high prevalence of hypertension in the group with low or no education in this study may be related to having a higher number of risk factors such as stress, poor dietary habits, poor working conditions, and lack of access to health services [50]. Urban residence increased the odds for hypertension in previous studies $[10,12,18]$ but in this study it was only significant in bivariate analysis among men and nonsignificant for women. It seems that the urban-rural differences in the prevalence of hypertension, as found in the 2007 IFLS [8], no longer exist in the 2013-14 IFLS in Indonesia.

Regarding weight variables, being underweight was protective and being overweight or obese increased the odds 


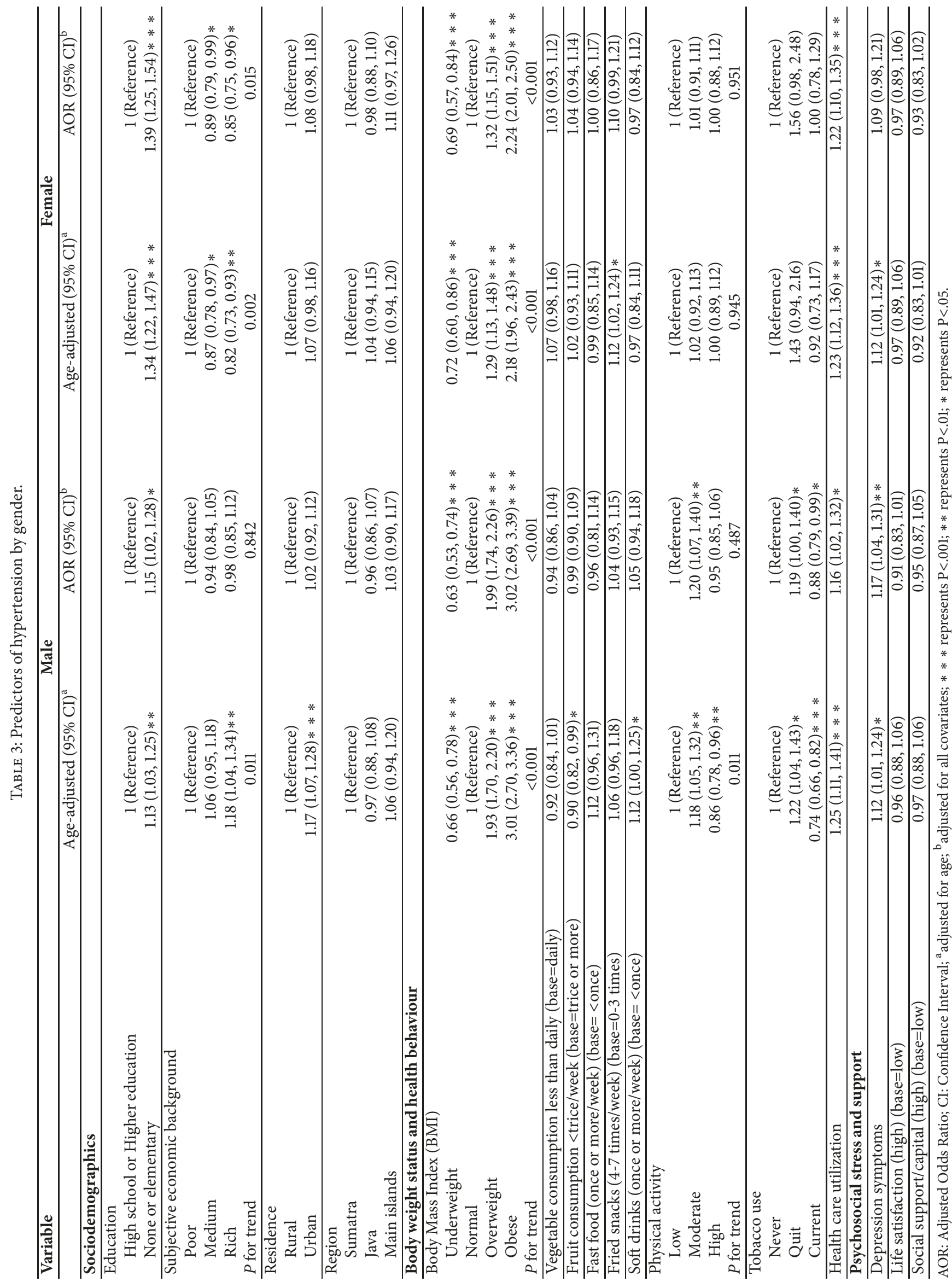


of having hypertension. The association between overweight/obesity and hypertension has been confirmed in a number of studies [8,10,13,15-17]. Overweight or obesity may be associated with hypertension independently but it could also be mediated through unhealthy diet and inadequate physical activity [14]. The negative association between being underweight and hypertension was also found in a study in Myanmar [10]. Some studies [e.g., [51]] suggest that about two-thirds of the prevalence of hypertension may be attributed to obesity.

As found in a number of previous studies [10, 13, 27-29], moderate physical activity was in this study among men but not among women negatively associated with hypertension. The elevated blood pressure among the female participants of the study may not be attributable to physical inactivity but linked to other factors such as obesity [52]. In agreement with some previous studies $[22,23]$, this study found that consumption of fatty foods (fried snacks) was among women associated with hypertension.

Unlike previous studies [19-21, 24-26], this study did not find an association between fruit and vegetable, soft drink, fatty foods (fried snacks), fast food consumption, and hypertension. Current smoking is a significant risk factor for hypertension [29, 30]. However, in this study among men only former tobacco use was positively associated with hypertension, while current tobacco use was negatively associated with hypertension. Among smokers in this study the number of cigarettes smoked per day (Mean=11.7) did not differ between hypertensives and nonhypertensives (Mean: 11.9) $(\mathrm{P}=0.150)$. It is possible that the impact of current tobacco use on hypertension is delayed, and, thus, current tobacco use may not be closely correlated with hypertension [53]. In a life-course impact of smoking on hypertension study, Gao et al. [53] found that current number of cigarettes smoked per day was negatively associated with the risk of hypertension; however, the increase of life-course-adjusted number of cigarettes smoked per day was associated with higher risks of hypertension.

The burden of having hypertension may also be expressed in the association between past 4 weeks outpatient health care utilization and hypertension, as also found in a previous study [31] of hypertension. In terms of psychosocial stress and support, this study found, in agreement with previous studies [32-34], that depression was among men (and in bivariate analysis among women) positively related to hypertension. Hyperreactivity of the sympathetic nervous system and genetic influences seem to be influencing mechanisms in the relationship between depression and hypertension [54]. Unlike previous studies [32, 35-37], this study did not find an association between low life satisfaction and lack of social support or capital and hypertension.

\section{Study Limitations}

Apart from anthropometric and blood pressure measurements, a study limitation was that all the other information assessed in this analysis was based on self-reporting. It is possible that certain behaviours were over- or underreported. Several essential determinants for hypertension such as serum fasting blood surge, Low-Density Lipoprotein (LDL), and total cholesterol (TC) were not assessed in this study and should be included in future studies. Further, it was a crosssectional study and causal relationships between risk factors and the development of hypertension can be established. Another limitation was that salt intake and heavy alcohol use, which are determinants of hypertension, were not assessed in this study.

\section{Conclusion}

The study found a high prevalence of hypertension in a representative sample of the general adult population in Indonesia. Less than half of hypertensives were aware and a minority were treated and controlled. Several risk factors, including sociodemographic variables (older age, lower education, being female, and lower socioeconomic status), body weight status (overweight or obese), health behaviour (physical inactivity), and psychosocial stress (depression), have been identified, which can help in guiding intervention programmes. Interventions programmes operating at multiple levels are urgently needed that can increase awareness of hypertension, access to high blood pressure treatment, and community wide health behaviour interventions that were identified and known to be effective in reducing high blood pressure levels. Future studies may want to include additional measures such as salt intake and alcohol consumption.

\author{
Abbreviations \\ BMI: Body mass index \\ DBP: Diastolic blood pressure \\ CES-D: Centres for Epidemiologic Studies \\ Depression Scale \\ EA: Enumeration area \\ IFLS: Indonesian Family Life Survey \\ IPAQ: International Physical Activity Questionnaire \\ LDL: Low-density lipoprotein \\ NCD: Noncommunicable disease \\ SBP: $\quad$ Systolic blood pressure.
}

\section{Data Availability}

The data underlying this study belong to the Indonesia Family Life Survey and are accessible via the RAND website: http://www.rand.org/labor/FLS/IFLS.html.

\section{Ethical Approval}

The IFLS has been approved by ethics review boards of RAND and University of Gadjah Mada in Indonesia [38].

\section{Consent}

Written informed consent was obtained from all respondents prior to data collection.

\section{Conflicts of Interest}

The authors declare that they have no conflicts of interest. 


\section{Authors' Contributions}

Karl Peltzer and Supa Pengpid conceived and designed the analysis. Karl Peltzer drafted the manuscript and Supa Pengpid made critical revision of the manuscript for key intellectual content. All authors read and approved the final version of the manuscript. All authors have agreed to authorship and order of authorship for this manuscript.

\section{Acknowledgments}

The research was conducted based on the IFLS-5 conducted by RAND (http://www.rand.org/labor/FLS/IFLS.html). We thank RAND for providing the access to the survey data and the study participants who provided the survey data.

\section{References}

[1] World Health Organization (WHO). Global status report on noncommunicable diseases 2014. Geneva, Switzerland: WHO, 2014.

[2] J. Schröders, S. Wall, M. Hakimi et al., "How is Indonesia coping with its epidemic of chronic noncommunicable diseases? A systematic review with meta-analysis," PLoS ONE, vol. 12, no. 6, p. e0179186, 2017.

[3] World Health Organization (WHO). Indonesia: WHO statistical profile, 2012. http://www.who.int/gho/countries/idn.pdf? ua $=1$.

[4] World Health Organization (WHO). Noncommunicable diseases country profiles 2014. http://apps.who.int/iris/bitstream/ 10665/128038/1/9789241507509_eng.pdf.

[5] M. M. Ibrahim and A. Damasceno, "Hypertension in developing countries," The Lancet, vol. 380, no. 9841, pp. 611-619, 2012.

[6] B. M. Egan, Y. Zhao, and R. N. Axon, "US trends in prevalence, awareness, treatment, and control of hypertension, 1988-2008," The Journal of the American Medical Association, vol. 303, no. 20, pp. 2043-2050, 2010.

[7] R. Castillo, "Prevalence and management of hypertension in Southeast Asia," J Hypertens, supplement 1, p. e4, 2016.

[8] M. A. Hussain, A. A. Mamun, C. Reid, R. R. Huxley, and Y. Li, "Prevalence, Awareness, Treatment and Control of Hypertension in Indonesian Adults Aged $\geq 40$ Years: Findings from the Indonesia Family Life Survey (IFLS)," PLoS ONE, vol. 11, no. 8, p. e0160922, 2016.

[9] C. Naing, P. N. Yeoh, V. N. Wai, N. N. Win, L. P. Kuan, and K. Aung, "Hypertension in Malaysia: An Analysis of Trends From the National Surveys 1996 to 2011," Medicine, vol. 95, no. 2, p. e2417, 2016.

[10] M. B. Bjertness, A. S. Htet, H. E. Meyer et al., "Prevalence and determinants of hypertension in Myanmar - a nationwide crosssectional study," BMC Public Health, vol. 16, no. 1, 2016.

[11] P. Katulanda, P. Ranasinghe, R. Jayawardena, G. R. Constantine, M. H. Rezvi Sheriff, and D. R. Matthews, "The prevalence, predictors and associations of hypertension in Sri Lanka: A cross-sectional population based national survey," Clinical and Experimental Hypertension, vol. 36, no. 7, pp. 484-491, 2014.

[12] P. T. Son, N. N. Quang, N. L. Viet et al., "Prevalence, awareness, treatment and control of hypertension in Vietnam-results from a national survey," Journal of Human Hypertension, vol. 26, no. 4, pp. 268-280, 2012.
[13] J. Wang, L. Zhang, F. Wang, L. Liu, and H. Wang, "Prevalence, awareness, treatment, and control of hypertension in China: results from a national survey," American Journal of Hypertension, vol. 27, no. 11, pp. 1355-1361, 2014.

[14] A. Esteghamati, M. Abbasi, S. Alikhani et al., "Prevalence, awareness, treatment, and risk factors associated with hypertension in the Iranian population: The national survey of risk factors for noncommunicable diseases of Iran," American Journal of Hypertension, vol. 21, no. 6, pp. 620-626, 2008.

[15] C. K. Chow, K. K. Teo, S. Rangarajan et al., "Prevalence, awareness, treatment, and control of hypertension in rural and urban communities in high-, middle-, and low-income countries," Journal of the American Medical Association, vol. 310, no. 9, pp. 959-968, 2013.

[16] S. Cuschieri, J. Vassallo, N. Calleja, N. Pace, and J. Mamo, “The Effects of Socioeconomic Determinants on Hypertension in a Cardiometabolic At-Risk European Country," International Journal of Hypertension, vol. 2017, Article ID 7107385, 7 pages, 2017.

[17] M. E. Hendriks, F. W. N. M. Wit, M. T. L. Roos et al., "Hypertension in Sub-Saharan Africa: cross-sectional surveys in four rural and urban communities," PLoS ONE, vol. 7, no. 3, Article ID e32638, 2012.

[18] C. Agyemang, "Rural and urban differences in blood pressure and hypertension in Ghana, West Africa," Public Health, vol. 120, no. 6, pp. 525-533, 2006.

[19] B. Li, F. Li, L. Wang, and D. Zhang, "Fruit and Vegetables Consumption and Risk of Hypertension: A Meta-Analysis," The Journal of Clinical Hypertension, vol. 18, no. 5, pp. 468-476, 2016.

[20] L. Wang, J. E. Manson, J. M. Gaziano, J. E. Buring, and H. D. Sesso, "Fruit and vegetable intake and the risk of hypertension in middle-aged and older women," American Journal of Hypertension, vol. 25, no. 2, pp. 180-189, 2012.

[21] D. Zhao, Y. Qi, Z. Zheng et al., "Dietary factors associated with hypertension," Nature Reviews Cardiology, vol. 8, no. 8, pp. 456465, 2011.

[22] C. Ezekwesili, C. Ononamadu, O. Onyeukwu, and N. Mefoh, "Epidemiological survey of hypertension in Anambra state, Nigeria," Nigerian Journal of Clinical Practice, vol. 19, no. 5, pp. 659-667, 2016.

[23] A. M. El-Badawy, H. M. Al-Kharusi, and S. A. Al-Ghanemy, "Health habits and risk factors among Omanis with hypertension," Saudi Medical Journal, vol. 26, no. 4, pp. 623-629, 2005.

[24] X. Xu, J. Byles, Z. Shi, P. McElduff, and J. Hall, "Dietary pattern transitions, and the associations with BMI, waist circumference, weight and hypertension in a 7-year follow-up among the older Chinese population: A longitudinal study," BMC Public Health, vol. 16, no. 1, article no. 743, 2016.

[25] S. Kar and B. Khandelwal, "Fast foods and physical inactivity are risk factors for obesity and hypertension among adolescent school children in east district of Sikkim, India," Journal of Natural Science, Biology and Medicine, vol. 6, no. 2, pp. 356-359, 2015.

[26] W. Cheungpasitporn, C. Thongprayoon, P. J. Edmonds et al., "Sugar and artificially sweetened soda consumption linked to hypertension: A systematic review and meta-analysis," Clinical and Experimental Hypertension, vol. 37, no. 7, pp. 587-593, 2015.

[27] M. Borjesson, A. Onerup, S. Lundqvist, and B. Dahlof, "Physical activity and exercise lower blood pressure in individuals with hypertension: Narrative review of 27 RCTs," British Journal of Sports Medicine, vol. 50, no. 6, pp. 356-361, 2016. 
[28] A. Giday and B. Tadesse, "Prevalence and determinants of hypertension in rural and urban areas of southern Ethiopia," Ethiop Med J, vol. 49, pp. 139-147, 2011.

[29] S. S. De Ramirez, D. A. Enquobahrie, G. Nyadzi et al., "Prevalence and correlates of hypertension: A cross-sectional study among rural populations in sub-Saharan Africa," Journal of Human Hypertension, vol. 24, no. 12, pp. 786-795, 2010.

[30] A. Leone, "Smoking and hypertension: independent or additive effects to determining vascular damage?" Current Vascular Pharmacology, vol. 9, no. 5, pp. 585-593, 2011.

[31] E. Macia, P. Duboz, and L. Gueye, "Prevalence, awareness, treatment and control of hypertension among adults 50 years and older in Dakar, Senegal," Cardiovascular Journal of Africa, vol. 23, no. 5, pp. 265-269, 2012.

[32] Y. Cuffee, C. Ogedegbe, N. J. Williams, G. Ogedegbe, and A. Schoenthaler, "Psychosocial risk factors for hypertension: an update of the literature," Current Hypertension Reports, vol. 16, no. 10, p. 483, 2014.

[33] M.-Y. Liu, N. Li, W. A. Li, and H. Khan, "Association between psychosocial stress and hypertension: a systematic review and meta-analysis," Neurological Research, vol. 39, no. 6, pp. 573580, 2017.

[34] A. T. Ginty, D. Carroll, T. J. Roseboom, A. C. Phillips, and S. R. De Rooij, "Depression and anxiety are associated with a diagnosis of hypertension 5 years later in a cohort of late middle-aged men and women," Journal of Human Hypertension, vol. 27, no. 3, pp. 187-190, 2013.

[35] V. Romero Martinez, E. Silva, and J. Villasmil, "Relationship between life satisfaction levels and high blood pressure in adolescents: PP.14.43," Journal of Hypertension, vol. 28, p. e260, 2010.

[36] S. Mojon-Azzi and A. Sousa-Poza, "Hypertension and life satisfaction: An analysis using data from the Survey of Health, Ageing and retirement in Europe," Applied Economics Letters, vol. 18, no. 2, pp. 183-187, 2011.

[37] M. S. Mujahid, A. V. D. Roux, and J. D. Morenoff, "Neighborhood characteristics and hypertension," Epidemiology, vol. 19, no. 4, pp. 590-598, 2008.

[38] J. Strauss, F. Witoelar, and B. Sikoki, The Fifth Wave of the Indonesia Family Life Survey (IFLS5): Overview and Field Report, vol. 1, RAND Corporation, 2016.

[39] A. V. Chobanian, G. L. Bakris, and H. R. Black, "Seventh report of the Joint National Committee on prevention, detection, evaluation, and treatment of high blood pressure," Hypertension, vol. 42, no. 6, pp. 1206-1252, 2003.

[40] C. P. Wen, T. Y. David Cheng, S. P. Tsai et al., "Are Asians at greater mortality risks for being overweight than Caucasians? Redefining obesity for Asians," Public Health Nutrition, vol. 12, no. 4, pp. 497-506, 2009.

[41] C. L. Craig, A. L. Marshall, and M. Sjöström, "International physical activity questionnaire: 12 -country reliability and validity," Medicine \& Science in Sports \& Exercise, vol. 35, no. 8, pp. 1381-1395, 2003.

[42] International Physical Activity Questionnaire (IPAQ). IPAQ Scoring Protocol. https://sites.google.com/site/theipaq/.

[43] E. M. Andresen, J. A. Malmgren, W. B. Carter, and D. L. Patrick, "Screening for depression in well older adults: evaluation of a short form of the CES-D (Center for Epidemiologic Studies Depression Scale)," American Journal of Preventive Medicine, vol. 10, no. 2, pp. 77-84, 1994.
[44] K. T. Mills, J. D. Bundy, T. N. Kelly et al., "Global disparities of hypertension prevalence and control: A systematic analysis of population-based studies from 90 countries," Circulation, vol. 134, no. 6, pp. 441-450, 2016.

[45] M. Hosseini, M. Yousefifard, M. Baikpour et al., "Twenty-year dynamics of hypertension in Iranian adults: age, period, and cohort analysis," Journal of the American Society of Hypertension, vol. 9, no. 12, pp. 925-934, 2015.

[46] World Health Organization (WHO). The Republic of Indonesia health system review. Health systems in transition. Vol-7, Number -1. New Dehli: WHO Regional Office for South-East Asia.

[47] S. Pengpid and K. Peltzer, "Overweight, obesity and associated factors among 13-15 years old students in the association of Southeast Asian Nations member countries, 2007-2014," Southeast Asian Journal of Tropical Medicine and Public Health, vol. 47, no. 2, pp. 250-261, 2016.

[48] K. Peltzer and S. Pengpid, "The Association of Dietary Behaviors and Physical Activity Levels with General and Central Obesity among ASEAN University Students," AIMS Public Health, vol. 4, no. 3, pp. 301-303, 2017.

[49] C. N. Rachmi, M. Li, and L. Alison Baur, "Overweight and obesity in Indonesia: prevalence and risk factors-a literature review," Public Health, vol. 147, pp. 20-29, 2017.

[50] C. Erem, A. Hacihasanoglu, M. Kocak, O. Deger, and M. Topbas, "Prevalence of prehypertension and hypertension and associated risk factors among Turkish adults: trabzon hypertension study," Journal of Public Health, vol. 31, no. 1, pp. 47-58, 2009.

[51] E. Afrifa-Anane, C. Agyemang, S. N. A. Codjoe, G. Ogedegbe, and A. De-Graft Aikins, "The association of physical activity, body mass index and the blood pressure levels among urban poor youth in Accra, Ghana," BMC Public Health, vol. 15, no. 1, 2015.

[52] R. M. Krauss, M. Winston, B. J. Fletcher, and S. M. Grundy, "Obesity: Impact on cardiovascular disease," Circulation, vol. 98, no. 14, pp. 1472-1476, 1998.

[53] K. Gao, X. Shi, and W. Wang, “The life-course impact of smoking on hypertension, myocardial infarction and respiratory diseases," Scientific Reports, vol. 7, no. 1, 2017.

[54] A. Z. Scalco, M. Z. Scalco, J. B. S. Azul, and F. Lotufo Neto, "Hypertension and depression.," Clinics (São Paulo, Brazil), vol. 60 , no. 3, pp. 241-250, 2005. 


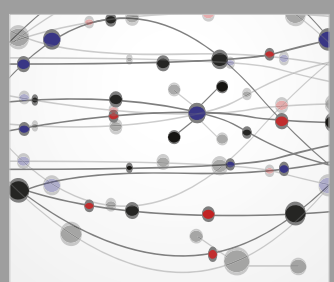

The Scientific World Journal
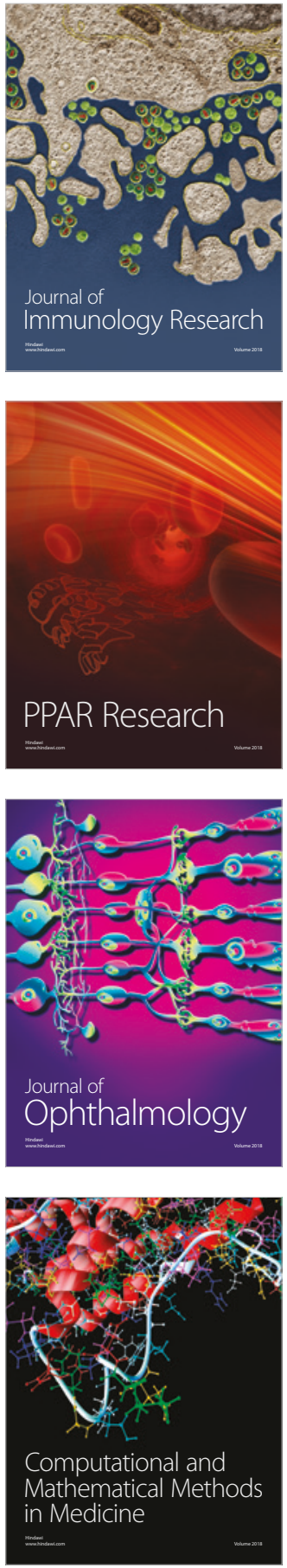

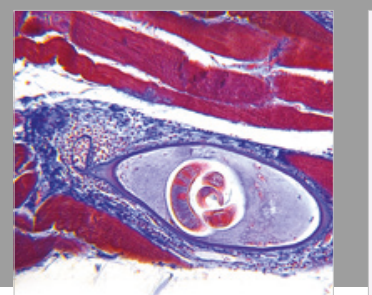

Gastroenterology Research and Practice

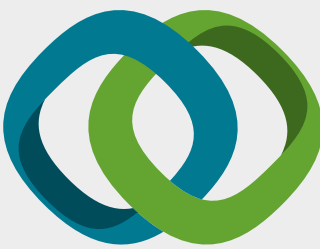

\section{Hindawi}

Submit your manuscripts at

www.hindawi.com
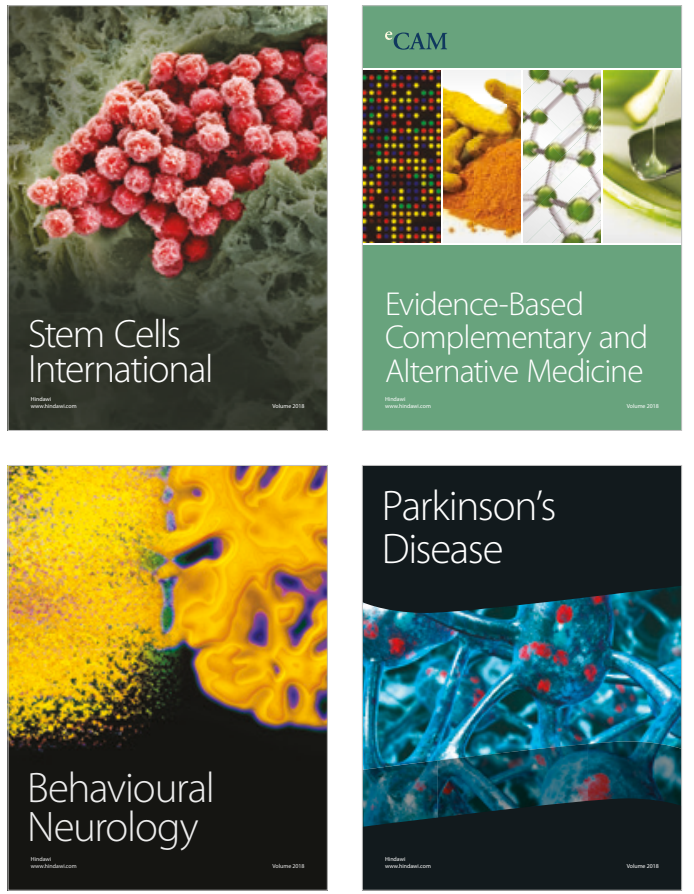

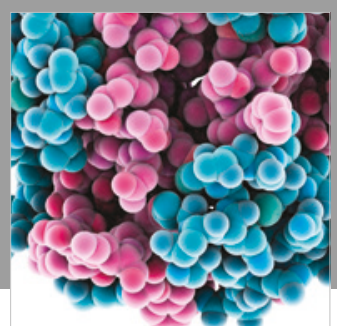

ournal of

Diabetes Research

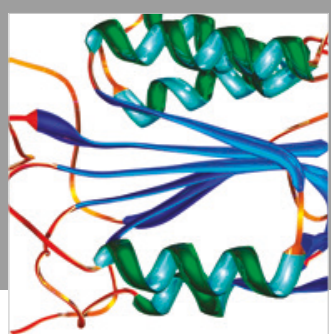

Disease Markers
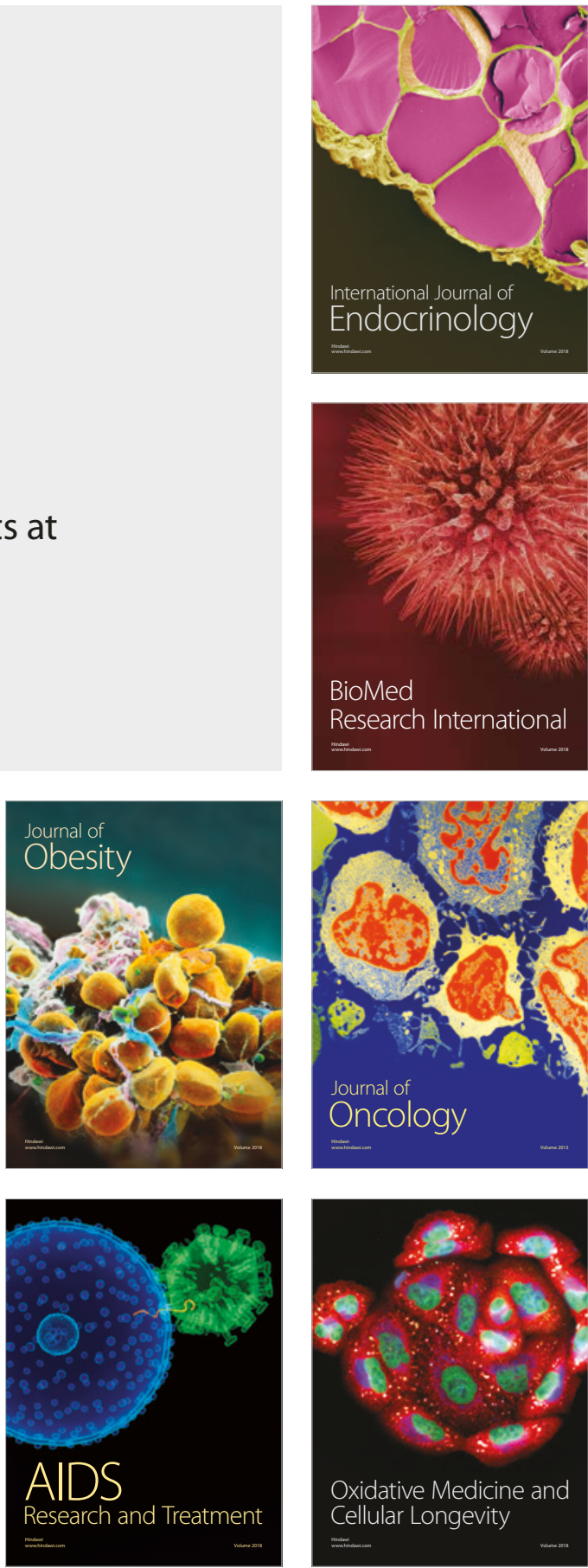